\title{
Glassy Dynamics of Driven Elastic Manifolds
}

\author{
Valerii M. Vinokur \\ Materials Science Division

\begin{abstract}
The submitted manuscript has been created by the University of Chicago as Operator of Argonne National Laboratory ("Argonne") under Contract No. W-31-109-ENG-38 with

the U.S. Department of Energy. The U.S.

Government retains for itself, and others acting on its behalf, a paid-up, nonexclusive, irrevocable worldwide license in said article to reproduce, prepare derivative works, distribute copies to the public, and perform publicly and display publicly, by or on behalf of the Government.
\end{abstract}

P.) 2010

Argonne National Laboratory, Argonne, IL 60439

Proceedings of the Conference: Landscape Paradigms in Physics and Biology, Los Alamos, NM, May 14-17, 1996, to be published in PHYSICA D.

Work supported by the U.S. Department of Energy, BES-Materials Sciences under contract \#W-31-109-ENG-38 and at ETH-Zurich by the Swiss National Foundation. 


\section{DISCLAIMER}

Portions of this document may be illegible in electronic image products. Images are produced from the best available original document. 


\title{
Glassy Dynamics of Driven Elastic Manifolds
}

\author{
Valerii M. Vinokur \\ Material Science Division, Argonne National Laboratory, Argonne, IL 60439 \\ (January 7,1997 )
}

We study the low-temperature dynamics of an elastic manifold driven through a random medium. For driving forces well below the zero-temperature depinning force, the manifold advances via thermally activated hops over the energy barriers separating favorable metastable states. We develop a scaling theory of the thermally activated dynamics (creep) and find a nonlinear glassy response for the driven manifold, $v \sim \exp \left(-\right.$ const $\left.\times F^{-\mu}\right)$. We consider an exactly solvable one-dimensional model for random driven dynamics which exhibits a creep-like velocity-force characteristic. We discuss a microscopic mechanism for the creep motion and show that the distribution of waiting times for the hopping processes scales as a power-law. This power-law distribution naturally yields an exponential response for the creep of the manifold.

key words: elastic manifold, disorder, creep motion, glassy response, algebraic distributions 
]

The dynamics of elastic manifolds in random media has become a central topic of modern statistical mechanics during the last decade and is in a state of rapid current development. The reasons for this interest are twofold. On one hand, elastic manifolds in a random environment are one of the simplest examples of a glassy system and yet exhibit a very rich static and dynamical behavior. Secondly, they represent a generic model for a study of both nonlinear collective transport in driven disordered systems, such as charge density waves, polymers, driven interfaces, dislocations in solids, and magnetic flux lines in type II superconductors, as well as stochastic kinetic processes, such as stochastic growth and kinetic roughening (see $[1,2]$ for a review).

The dynamics of driven elastic manifolds is the result of the interplay between quenched disorder and the interaction among the many elastic degrees of freedom that compose the manifold. A key physical quantity describing the dynamics is the average velocity $v$ of the driven manifold as a function of the applied force $F$. At zero temperature there is a depinning transition from a pinned state where $v=0$ to a sliding state at a critical driving force $F_{c}$. A finite temperatures washes out the transition and the mean velocity is then finite for all driving forces. For low temperatures and driving forces well below the $T=0$ depinning threshold, $F_{c}$, the dynamics of the driven manifold is controlled by thermally activated jumps of correlated regions over the pinning energy barriers separating different metastable states. In this region the mean velocity is highly nonlinear and has been evaluated via a scaling approach $[3,4]$ with the result,

$$
v \simeq \exp [-U(F) / T]
$$

where $U(F)$ is the optimal energy barrier for creep between favorable metastable states. Under the action of the external drive, sections of the manifold that are initially pinned move to a more favorable metastable state, determined by the condition that the energy gain due to the driving force equals the elastic deformation and pinning energy of the medium. For $F<<F_{c}$ large sections of the manifold hop long distances to find the next optimal-energy state. This yields a large energy barrier that diverges algebraically with vanishing driving force,

$$
U(F) \sim\left(F_{c} / F\right)^{\mu},
$$

with $\mu$ a characteristic exponent. The form given in Eqs. (1),(2) was first proposed for the motion of dislocations in crystals [4] and is now widely used to describe the low temperature dynamics of vortex lines in type-II superconductors [1].

An important recent development has been the confirmation by numerical studies of an elastic string in $d=2$ of the basic assumption of the scaling approach [4] that the barriers between the metastable states scale with the length of the fluctuating string segment in the same way as the fluctuations in the free energy (aside from logarithmic corrections) [5]. Despite a substantial analytical [6-8] and numerical [9-14] study of the dynamics of driven disordered elastic manifolds, many open questions remain.

In this paper we describe a scaling approach to the driven manifold dynamics developed first in the context of dislocation motion [4], then consider a simple exactly solvable one dimensional model for driven dynamics in random environment, which in spite of its simplicity captures an essential feature of the non-linear manifold motion, and in conclusion we discuss the nature of the mechanism by which the manifold selects the appropriate optimal segment controlling the dynamics.

\section{SCALING APPROACH.}

The Hamiltonian of a $D$-dimensional elastic manifold driven through a $d$-dimensional disordered medium is

$$
\mathcal{H}=\int d^{d} x\left[\frac{C}{2}\left(\frac{\partial \mathbf{u}}{\partial \mathbf{x}}\right)^{2}+U(\mathbf{x}, \mathbf{u})-\mathbf{F} \cdot \mathbf{u}\right],
$$

where $C$ denotes an elastic stiffness constant (e.g., the linear tension of the string; for simplicity we consider the isotropic medium) and $\mathbf{u}(\mathbf{x}, t)$ is the $n$-dimensional transverse displacement field of the manifold. Eq. (3) describes for instance an elastic string $(D=1)$ in two $(d=2, n=1)$ and three $(d=3, n=2)$ dimensions, and two- or 
three-dimensional vortex lattices ( $d=2, D=2, n=2$ and $d=3, D=3, n=2$, respectively). The disorder consists of uncorrelated point defects of density $n_{\boldsymbol{i}}$. It is described by a random potential $U(\mathbf{x}, \mathbf{u})$ of range $\xi$ and variance $\Delta=v_{0} \sqrt{n_{i} \xi^{d}}$, with $v_{0}$ the maximum depth of the potential well of a single pinning center. In the absence of driving force the string adjusts to the random landscape and traverses the medium along rough optimal paths determined by balancing the elastic and pinning energies. The geometry of these optimal paths is characterized by the roughness of the manifold, defined as $w(L)=\left\langle[\mathbf{u}(\mathbf{x}+\mathbf{L})-\mathbf{u}(\mathbf{x})]^{2}\right\rangle^{1 / 2}$, where $\langle\ldots\rangle$ denotes the average over both thermal fluctuations and quenched disorder. At large distances the roughness scales as $w \approx \xi\left(L / L_{c}\right)^{\zeta}$, where $\zeta<1$ is the roughness exponent and $L_{c}$ is the pinning correlation length. We consider the case of weak disorder such that $L_{c} \gg \xi$. In this regime domains of linear size $L_{c}=\xi\left(C \xi^{D} / \Delta\right)^{2 /(4-D)}$ are pinned coherently by disorder when $F=0$. The pinning length $L_{c}$ is the smallest scale on which barriers between metastable states exist at $F=0$. The minimum average energy barrier between neighboring metastable positions of a pinned segment $L_{c}$ is $U_{c}=C \xi^{2} / L_{c}^{(D-2)}$. Optimal metastable configurations of sizes $L>L_{c}$ are then separated by barriers $U(L) \simeq U_{c}\left(L / L_{c}\right)^{2 \zeta+D-2}$. At $T=0$ the string starts to slide when the applied force $F$ can depin a region of linear size $L_{c}$, yielding a threshold force for sliding $F_{c}=C \xi / L_{c}^{2}$.

We now consider the dynamics at a finite but low temperature $T \ll U_{c}$, where the elementary pinning scales $U_{c}$ and $L_{c}$ are not renormalized significantly by thermal fluctuations [1]. Under the action of the driving force a domain of size $L$ can be displaced to a new more favorable metastable state. For $F \ll F_{c}$ the dynamics can be described as the nucleation of an elementary excitation or nucleus of size $L$. The free energy cost for creating such a nucleus is given by $\mathcal{F}[L]=U_{c}\left(L / L_{c}\right)^{2 \zeta+D-2}-F L_{c}^{D} \xi\left(L / L_{c}\right)^{\zeta+D}$. The size $L_{\text {opt }}$ of the optimal excitation is obtained by minimizing this free energy cost, with the result $L_{\text {opt }}=L_{\mathrm{c}}\left(F_{\mathrm{c}} / F\right)^{1 /(2-\zeta)}$. Nuclei with $L<L_{\text {opt }}$ collapse, while nuclei of size $L>L_{\text {opt }}$ expand. In other words excitations on scales larger than $L_{\text {opt }}$ slide freely, while pinning barriers on scales $L<L_{\text {opt }}$ can be overcome only via thermally activated hops. The length scale $L_{\text {opt }}$ determines the upper bound above which thermally activated processes are no longer relevant. The creep rate is determined by the corresponding optimal energy barrier, $U(F)=\mathcal{F}\left(L_{\text {opt }}\right)$, with $U(F)=U_{c}\left(F_{c} / F\right)^{\mu}$, where $\mu=(2 \zeta+D-2) /(2-\zeta)$. For an elastic string in two dimensions $\zeta=2 / 3, D=1$, and $\mu=1 / 4$.

\section{ONE DIMENSIONAL MODEL}

The above qualitative picture of the low temperature creep motion $[4,1]$ is based on dimensional scaling arguments and numerical simulations. A rigorous analysis is still lacking and a general analytical derivation for the relevant physical models remains an unsolved problem. In the absence of a rigorous analysis of realistic physical situations one is seeking for models which are simple enough to be treated analytically and yet are able to mimic the large diversity of dynamics of real disordered systems. A well known example is the problem of a single particle driven by an external force $f$ and subject to a one dimensional random force field with Gaussian short range correlations. The term random force means that the correlator of the random potential $U(x)$ is $\overline{(U(x)-U(y))^{2}} \sim \Delta|x-y|$ where $\Delta$ characterizes the strength of the random potential. This model is known as Sinai's model and has long been a subject of extensive studies [15-18,3,19-21] The remarkable result obtained for this model is that even at finite temperature the mobility vanishes below a threshold force $f_{t h} \sim \Delta / 2 T$. Moreover this system was found to exhibit anomalous diffusion, and aging phenomena [20] very much like what is observed in spin glasses [22,23]. We investigate a more general one-dimensional model with a Gaussian random potential having correlator of the form [24]:

$$
K(x-y) \sim \Delta\left(\left(\frac{|x-y|}{\xi_{G}}\right)^{\gamma}+\log \left(\frac{|x-y|}{a}\right)\right.
$$

Tuning the parameter $\gamma$ we can cross over from the Sinai's case $(\gamma=1)$, to glassy creep dynamics at $0<\gamma<1$ (see below) and also mimic the transition from the glassy to viscous flow dynamics at $\gamma<0$. Note that the latter transition mimics the glass transition taking place, for example at the vortex lattice melting [1], where the elasticity of the manifold (vortex lattice in this case) breaks down result in the disappearance of the long correlations in the effective random potential. The first term which dominates at large distance describes long range correlations in the random potential and generalizes Sinai's model. The second term describes the behavior at the critical point $\xi_{G}=\infty$. Indeed one has $\gamma=0$ and $\tilde{K}(q) \sim 1 / q$ at the transition and thus $K(x) \sim \Delta \ln x$. The form (4) is an interpolation resulting from the crossover between the critical fixed point and the fixed point describing the glass phase. In the uncorrelated phase correlations are short range and one chooses a correlator as $\tilde{K}(q)=1 / \sqrt{q^{2}+\left(\xi_{G}\right)^{-2}}$, i.e $K(x)=K_{0}(x / \xi)$, so as to reproduce the critical behavior for $a \ll x \ll \xi_{G}$. An identical scenario was demonstrated 
using RG for the correlations in the free energy landscape at the glass transition in surface growth models, such as the directed polymer in $d \geq 2+1[26]$

On a mathematical level the present model is the $d=0, n=1$ version of the problem of the dynamics of manifolds of internal dimension $d$, in a $n$ dimensional space. Remarkably, the case $d=0$ and $n \rightarrow \infty$ was recently studied by completely different techniques and seems to exhibit similar regimes.

We consider the Langevin diffusion in the one dimensional quenched random potential $U(x)$ in presence of a global bias $f$ and thermal white noise $\eta(t)$ :

$$
\frac{d x(t)}{d t}=-\nabla U(x(t))+F+\eta(t)
$$

with $\left\langle\eta(t) \eta\left(t^{\prime}\right)\right\rangle=2 T \delta\left(t-t^{\prime}\right)$ and $T$ is the temperature. The probability density $P(x, t)$ and the current $J(x, t)$ satisfy:

$$
\frac{\partial P(x, t)}{\partial t}=-\nabla J(x, t)
$$

with $J(x, t)=-T \nabla P(x, t)+(F-\nabla U(x)) P(x, t)$.

To derive the analytic expression for the velocity $v$ we generalize to continuum models the method introduced by Derrida [18] for discrete hopping problems. We consider an infinite periodic environment, i.e a periodic random force $\nabla U(x)$, of period $L$. The limit $L \rightarrow \infty$ is taken at the end. One defines the periodized probability $\tilde{P}(x)=\sum_{k} P(x+k L)$ which obeys the same equation (6) as $P$, and corresponds to diffusion on a periodic ring of size $L$. Using (6) the velocity for the particle on the infinite line can be expressed as:

$$
\begin{aligned}
\frac{d<x(t) \geq}{d t}= & -\int_{-\infty}^{+\infty} d x x \nabla J=\int_{-\infty}^{+\infty} d x J(x, t) \\
& =\int_{0}^{L} d x \tilde{J}(x, t)
\end{aligned}
$$

where $\tilde{J}(x, t)=-T \nabla \tilde{P}(x, t)+(F-\nabla U(x)) \tilde{P}(x, t)$. At long time $\tilde{J}(x, t)$ goes to a constant $\tilde{J}$ and the asymptotic velocity $V$ is exactly given by $v=\tilde{J} L$. To find $\tilde{J}$ for a fixed $L$ and disorder configuration one must solve the stationarity equation:

$$
T \frac{\partial \tilde{P}(x)}{\partial x}+(\nabla U(x)-F) \tilde{P}(x)=-\tilde{J}
$$

with the two additional conditions $\tilde{P}(0)=\tilde{P}(L)$ and $\int_{0}^{L} d x \tilde{P}(x)=1$. The stationary solution with zero current $\tilde{J}=0$, i.e the Gibbs distribution $P_{0}(x)=\exp \left(\frac{1}{T}(-U(x)+F x)\right)$ does not, in general, satisfy the periodic boundary conditions. Thus $v$ can be found from the solution with non-zero current:

$$
\begin{aligned}
\tilde{P}(x)= & \frac{\tilde{J}}{T}\left(\frac{\int_{0}^{L} d y e^{(U(y)-U(x)+(x-y) F) / T}}{1-e^{(U(L)-U(0)-F L) / T}}\right. \\
& \left.-\int_{0}^{x} d y e^{(U(y)-U(x)+(x-y) F) / T}\right)
\end{aligned}
$$

$\tilde{J}$ and thus $v$ follow from the normalization condition for $\tilde{P}$. In the limit $L \rightarrow \infty$, imposing the restriction $U(0)=U(L)$, unimportant for $F>0,(9)$ simplifies to:

$$
\tilde{P}(x)=\frac{\tilde{J}}{T} \int_{0}^{+\infty} d z e^{(U(x+z)-U(x)-F z) / T}
$$

and one gets the general formula for $v$ :

$$
\frac{1}{v}=\frac{1}{T} \int_{0}^{+\infty} d z e^{-F z / T}\left\langle e^{(U(x+z)-U(x)) / T}\right\rangle_{x}
$$

valid for an arbitrary potential $U(x)$. $\langle A\rangle_{x}$ denotes the translational average $\langle A\rangle_{x}=\lim _{L \rightarrow \infty} L^{-1} \int_{0}^{L} d x A(x)$. The average in (11) exists quite generally and is independent of the configuration of the random potential, i.e the velocity is self-averaging. The physical interpretation of (11) in terms of Arrhenius waiting time is transparent. The average 
waiting time $1 / v$ is a sum of Boltzman weights associated with the barriers the particle must overcome to move in the direction of the driving force. The highest barriers $U(x+z)-U(x)$ with $z>0$, produce the largest waiting times.

One can immediately verify that the expression (11) describes correctly the behavior in the limiting cases. At large $F$ one has $v \approx F$. On the other hand one expects the linear response at small forces, $F \rightarrow 0$, provided the random field is uncorrelated at large distances. The latter means that $\left\langle e^{(U(x+z)-U(x)) / T}\right\rangle \rightarrow\left\langle e^{U / T}\right\rangle\left\langle e^{-U / T}\right\rangle$ when $z \rightarrow \infty$, then:

$$
v \propto \frac{D}{D_{0}} F=\frac{F}{\left\langle e^{U / T}\right\rangle\left\langle e^{-U / T}\right\rangle}
$$

where $D$ and $D_{0}$ are the diffusion coefficients in the presence and in the absence of disorder, respectively and the Einstein relation holds. When $D \ll D_{0}$ the $v-F$ curve will show strong nonlinearity at intermediate scales where the transition between low force and high force regime of motion occurs.

We now turn to a detailed study of gaussian disorder with correlator $K(x)$. Upon averaging over disorder (11) yields:

$$
\frac{1}{v}=\frac{1}{T} \int_{0}^{\infty} d x \exp \left(-\frac{F x}{T}+\frac{K(x)}{2 T^{2}}\right)
$$

The choice of $K(x)$ as in (4) gives rise to several regimes of particle dynamics depending on the range of the correlations of the random potential. Our expression immediately reproduces Sinai's case $\gamma=1$ : for $\gamma>1$ the integral in (13) diverges and the velocity is zero. Sinai's model corresponds to $\gamma=1$ and appears as a marginal case where the integral (13) diverges for $F<F_{t h}=\Delta /\left(2 T \xi_{G}\right)$ and $v=0$ while $v=F-F_{t h}$ exactly for $F>F_{t h}$, in agreement with previous results $[15-17,19,20]$. The system with $\gamma=1$ exhibits algebraic distributions of waiting times which gives rise to aging phenomena [22]. Therefore Sinai's model mimics essential aspects of the spin-glass behavior. Interestingly, this $v$ versus $F$ dependence mimics also the dry friction phenomenon.

Now we show that the choice $0<\gamma<1$ gives rise to the creep motion. Defining the dynamical exponent $z=$ $2+\left(\Delta / 2 T^{2}\right)$ and the characteristic force $F_{c}=\left(\Delta / 2 T^{2}\right)^{1 / \gamma} T / \xi_{G}$ one arrives at:

$$
\begin{aligned}
& \frac{T}{v}=\frac{1}{F_{c}{ }^{z-1}} H\left(F / F_{c}\right) \\
& \quad H(f)=\int_{0}^{\infty} d q q^{z-2} \exp \left(-f q+q^{\gamma}\right)
\end{aligned}
$$

Note that $F_{c}$ reduces to the threshold force $F_{t h}$ when $\gamma \rightarrow 1$. At $\gamma<1$ the sharp threshold disappears but at $F \ll F_{c}$ the $v$ versus $F$ dependence shows strongly nonlinear behavior with an essential singularity at small $F$. Using the steepest descent method at $F \ll F_{c}$ one finds:

$$
\begin{gathered}
v=A T F_{c}^{z-1}\left(\frac{F}{F_{c}}\right)^{\frac{z-1-(\gamma / 2)}{1-\gamma}} \exp \left(-(1-\gamma)\left(\frac{\gamma F_{c}}{F}\right)^{\mu}\right), \\
\mu=\frac{\gamma}{1-\gamma}
\end{gathered}
$$

with $A \sim \sqrt{\gamma(1-\gamma) / 2 \pi}$. The exponential factor holds for any correlator behaving as a power law $\sim x^{\gamma}$ at large distances. The preexponential factor depends on details of the crossover of the correlator to the logarithmic regime. In this creep regime the linear response at $F \rightarrow 0$ is absent and the characteristic barriers which control the dynamics diverge as $1 / F^{\mu}$.

\section{MICROSCOPIC DESCRIPTION}

We now show that the form given in Eq. (1) for the mean motion arises naturally when the driven manifold sequentially nucleates all possible excitations on length scales $L<L_{\text {opt }}$ to overcome all the possible energy barriers separating metastable states on scales $E<U(F)$. For the sake of simplicity we consider an elastic string in $2 d$. We demonstrate that the distribution of waiting times $\tau(E)$ for hops between metastable states separated by energy barriers $E<U(F)$ scales as a power-law,

$$
\Psi(\tau) \simeq \tau^{-1-\alpha}
$$


with $\alpha<1$. This microscopic mechanism of string dynamics can be viewed as an avalanche-like motion. The distribution of waiting times given in (16) is cutoff at the waiting time $\tau[U(F)]$ corresponding to the optimal barrier $U(F)$. For hopping times $\tau$ exceeding $\tau[U(F)]$ only very rare barriers with $E \gg U(F)$ can retard the motion (rare events) and the distribution function decays much faster than in Eq. (16). The average waiting time that controls the mean string velocity is therefore determined by the cutoff $\tau[U(F)]$.

We begin by considering a segment of finite length $L<L_{\text {opt }}$ initially pinned in a metastable configuration. We derive an expression for the probability $P_{L}(E)$ that this segment encounters energy barriers smaller than $E$ when it samples all possible departures from its initial configuration under the action of the external force $F$. For any finite driving force the set of all configurations that the segment may sample during its activated motion form a connected cluster of temporarily pinned states. To describe this cluster, we fix the ends of the segment $L$ and imagine dividing it in elementary units of longitudinal size $L_{c}$. An elementary move consists of the hop of the $i$-th unit $L_{c}$ across a transverse distance $\xi$ to a new metastable position by overcoming the elementary pinning energy barrier $U_{i}$. Here the $\left\{U_{i}\right\}$ 's are random variables fluctuating around their average value, the minimal average pinning barrier, $U_{c}$. The segment $L$ can be thought of as a singly-connected necklace of $n$ (unit) beads, where the beads corresponds to the elementary sections $L_{c}$. To begin, we consider the simplest class of departures of $L$ from its initial configuration and describe the advance of $L$ via the hopping of individual beads over the fluctuating barriers $U_{i}$. The barrier associated with the motion of the segment $L$ is then the largest of the barriers of the elementary hops $L_{c}$. To understand this we note that the hop of a section $L$ of the string to a nearby metastable state can be thought of as the motion of the domain boundary between spin up and spin down regions in an Ising spin system with random couplings $\left\{J_{i}\right\}$. Each elementary unit $L_{c}$ corresponds to a single Ising spin. The hop of the $i$-th segment $L_{c}$ over the corresponding energy barrier $U_{i}$ corresponds to a single spin flip. The flip of spin $i_{0}$ along the domain boundary will cost an energy of order $J_{i_{0}-1}+J_{i_{0}+1}$, where $J_{i_{0}-1}$ and $J_{i_{0}+1}$ are the couplings to the neighboring spins. The flipping of this particular spin will facilitate the flipping of the neighboring ones, since now overturning spin $i_{0}-1$ will only cost an energy $J_{i_{0}-2}$, which may be larger or smaller than $J_{i_{0}-1}+J_{i_{0}+1}$. In either case the process of overturning the entire domain boundary will be controlled by the largest energy cost for flipping a single spin. If $L$ is composed of $n$ elementary segments $L_{c}$ with energy barriers $U_{i}$ randomly fluctuating about $U_{c}$, then the energy barrier for the segment $L$ is $U=\max U_{i}$. It is important to stress here that we are not trying to find the optimal hop for the segment $L$ and the associated optimal energy barrier, but we are simply discussing the distribution of all possible hops of the segment $L$ from the initial to all available final metastable states. In order to construct all possible configurations of the advancing segment, we now imagine redefining the network of elementary hops by choosing a new unit $L_{1}>L_{c}$ so that the section $L$ is now composed of, say, $n_{1}$ sub-blocks of size $L_{1}$ and fluctuating energy barriers $U_{1 i}$. The set of all possible configurations of the excitation $L$ as it visits all available metastable states forms a new cluster of elementary excitations or subclusters $L_{1}$. Notice that the subclusters of length $L_{1}$ may in general be multiply connected, bit the argument given above will still apply upon redefinition of the elementary unit. The global barrier for the motion of the new cluster will again be the maximum of the energy barriers of each subcluster. The linear structure of the string ensures that the cluster of all possible configurations of $L$ remains singly-connected at all levels of rescaling. It is precisely this linear topology that enables us to carry out this rescaling procedure. Repeated application of this procedure will allow us to list all possible configurations of the segment in questions and yield the limiting distribution that we seek. Such a distribution, if it exists, must be stable under the max operation and therefore belongs to the class of so-called extreme distributions [27]. To briefly summarize the theory of extreme distributions, we consider a set of identically distributed independent random variables $X_{i}$, with $1 \leq i \leq n$ - in the present problem these are all the energy barriers of the elementary spins and/or block of spins composing the segment $L$. Let $M_{n}=\max \left\{X_{1}, \ldots, X_{n}\right\}$. If $F(x)$ is the probability of the event $X_{i}<x$ and $\mathcal{P}_{n}(x)$ the probability that $M_{n}<x$, it can be shown that in the limit of large $n$ the probability distribution of the maxima can be approximated by an asymptotic form $\mathcal{P}(x)$, given by the solution of the functional equation $\lim _{n \rightarrow \infty} \mathcal{P}_{n}\left(x+a_{n}\right)=\lim _{n \rightarrow \infty} F^{n}\left(x+a_{n}\right)=\mathcal{P}(x)$, where $\mathcal{P}(x)$ is defined for $-\infty<x<\infty$. The extreme distribution we seek has the form $\mathcal{P}(x)=\exp [-\exp (-x)]$ [27]. The functional form of the distribution must remain invariant when the elementary unit of the cluster of all possible hops is redefined according to the procedure described earlier. Since $[\mathcal{P}(x)]^{n}=\exp [-n \exp (-x)]$, this requires $a_{n}=\ln n$. It thus follows that for the problem of interest here the appropriate variable $x$ is $E-\ln L$, where we measure $E$ in units of $U_{c}$ and $L$ in units of $L_{c}$. The probability distribution of energy barriers for a given segment $L$ is then $\mathcal{P}_{L}(E)=\exp [-L \exp (-E)]$. The corresponding distribution density $p_{L}(E)=d \mathcal{P}_{L}(E) / d E$ is

$$
p_{L}(E)=L e^{-E} \exp [-L \exp (-E)]
$$

The typical barrier of a segment of length $L$ scales then as $E \sim U_{c} \ln L$. The global distribution density $W(E)$ of energy barriers for the string is obtained by integrating $p_{L}(E)$ over $L$ with the proper weight $n_{L}$ describing the 
density of the segments of length $L$. At the critical point $F=0$, the cluster-sizes distribution is $n_{L} \sim L^{-\nu}$. At finite forces $F$ the same distribution will describe the cluster network up to $L=L_{\text {opt }}$. For the usual percolation clusters $\nu=1+d / d_{f}$, with $d$ the dimensionality of the medium and $D<d_{f}<d$ the fractal dimensionality of the clusters network [25]. For anisotropic directed percolation describing the depinning transition the exponent may be different and will be discussed elsewhere, but the relation $\nu>2$ holds. By evaluating the integral we find

$$
W(E) \sim e^{-(\nu-1) E / U_{c}} .
$$

The corresponding probability density of finding a waiting time $\tau=\tau_{0} \exp (E / T)$, with $\tau_{\mathrm{o}}$ a microscopic time scale, is given by

$$
\Psi(\tau) \sim T\left(\tau_{0} / \tau\right)^{1+\alpha}, \quad \alpha=(\nu-1) T / U_{c} .
$$

The distribution of energy barriers given in Eq. 18 must be cut off at the energy $U(F)$ corresponding to the optimal segment $L_{\text {opt }}(F)$ since the segments of larger scales are, on average, sliding freely and do not participate in the activated dynamics. The notion of "waiting time" makes no sense for such excitations. The average waiting time controlling the motion is therefore

$$
\langle\tau\rangle \sim \int^{\tau_{\max }} d \tau \Psi(\tau) \tau \sim \exp [(1-\alpha) U(F) / T]
$$

where $\tau_{m a x}=\tau_{0} \exp [U(F) / T]$. Now, since the motion is controlled by the largest barrier, one arrives at the average velocity given by $v \simeq u_{o p t} /\langle\tau\rangle \simeq \exp [-(1-\alpha) U(F) / T]$, recovering the result of the scaling theory [4].

We now discuss some observable consequences of the result given in Eq. 19. The vortex lattice in the mixed state of high temperature superconductors is probably the best system for the experimental study of different aspects of glassy dynamics, since by tuning parameters such as the applied field one can probe different regimes. For a driven vortex lattice the avalanche-like low temperature dynamics just described will manifest itself in the spectral density of the density-density correlation function, $S(\mathbf{k}=0, t)=\left\langle\delta n_{v}(\mathbf{r}, t) \delta n_{v}(\mathbf{r}, 0)\right\rangle$, which is accessible by Hall-probe measurements. The density-density correlation function can be expressed in terms of the correlation function of the displacement field $u(\mathbf{r}, t)$ as $S(\mathbf{k}, t)=k^{2}\langle u(\mathbf{k}, t) u(-\mathbf{k}, 0)\rangle$. On large distances displacements are uncorrelated in a glassy system and $\langle u(\mathbf{k}, t) u(-\mathbf{k}, 0)\rangle \propto\left(1 / k^{2}\right) f_{u}(\mathbf{k}, t)$. The corresponding spectral density $f_{u}(\omega)$ can be expressed in terms of the distribution function of energy barriers $W(E)$,

$$
f_{u}(\omega) \propto \int d E W(E) \frac{\tau(E)}{1+[\tau(E) \omega]^{2}}
$$

Using $W(E)$ from (18) we obtain a power law density noise spectrum,

$$
S(\omega) \propto \frac{1}{\omega^{1-\alpha}}
$$

We see that at low temperatures, $T<U_{c}$, the exponent $\alpha \ll 1$, and the noise spectrum of vortex density fluctuations is nearly the $1 / f$ spectrum.

We now turn to discuss the high temperature region, where thermal fluctuations renormalize the pinning energy barriers. The characteristic temperature $T_{d p}$ separating the high temperature and low temperature regions is defined by the self-consistent equation $T_{d p} \simeq U_{c}\left(T_{d p}\right)$ [1]. For elastic manifolds with $D \geq 2, U_{c}(T)$ grows much faster than $T$ [1] for $T>T_{d p}$, and $\alpha<1$. The results obtained earlier are therefore still relevant, with suitably renormalized parameters.

The situation is more subtle for the case of an elastic string, corresponding to $D=1$. Above the string depinning temperature, $T_{d p}^{s}$, the minimal pinning energy of the string becomes of order $T$, i.e., $U_{c}(T) \simeq T[4,1]$. The definition of the depinning temperature used here follows from a scaling theory based on the postulate that the creep barriers scale in the same way as the fluctuations in the free energy, i.e., the string statistical mechanics is controlled by a unique energy scale [4,5]. Such a scaling theory leaves numerical constants undetermined. To preserve the idea that a single energy scale controls the dynamics we define $T_{d p}^{s}$ as the temperature where first $\alpha(T)=1$. This definition avoids the introduction of a new unphysical energy scale depending on the fractal dimensionality of the cluster of the pinned states. For $T>T_{d p}^{s}$ the motion of the string on scales $L<L_{o p t}$ is still governed by the waiting times distribution function of Eq. 19 with $\alpha=1$, i.e., $\Psi(\tau) \sim\left(1 / \tau^{2}\right)$. The average waiting time in this high temperature regime is then 


$$
\bar{\tau}_{=} \tau_{0} \ln \left(\tau_{\max } / \tau_{0}\right) \sim F^{-\mu} .
$$

The characteristic energy and length of the segment hopping with this waiting time are $\bar{E} \simeq T \ln \left(\bar{\tau} / \tau_{0}\right) \sim \ln F$ and $\bar{L}=L_{c}\left(\bar{\tau} / \tau_{0}\right)$, respectively. The typical transverse displacement $\bar{u}$ of a length $\bar{L}$ of string is determined by $C \bar{u}^{2} / \bar{L} \simeq F \bar{u} \bar{L}$. Defining the string velocity as $v=\bar{u} / \bar{\tau}$ and recalling that the activated velocity contains a prefactor $\sim F$ ensuring that lux flow is recovered for $F>F_{c}$, we obtain

$$
v \sim\left(F / F_{c}\right)^{2-\mu}, T>T_{d p}^{s} .
$$

In the high temperature region, $T>T_{d p}^{s}$, the string velocity vanishes as a power law. This represents a marginal glassy dynamics which may be relevant to dislocation dynamics or for vortex creep in the high temperature, low magnetic field region of type II superconductors. We conjecture that this marginal dynamics corresponds to a new glassy state which can be referred to as critical glass.

In conclusion, we have presented a scaling approach describing thermally activated dynamics (creep) of elastic manifolds in quenched random environment. We have investigated a one dimensional exactly solvable model for the driven random dynamics and showed that the system exhibits strongly nonlinear creep-like response for the longrange correlated random field. We have developed a microscopic model for the glassy dynamics of elastic manifolds and found the exponential distribution of energy barriers characterizing the rugged energy landscape for elastic manifolds. the low-temperature creep is governed by a power law distribution of waiting times. The dynamics at low temperature, $T<T_{d p}$, and long times is dominated by the optimal barrier $U(F)$, corresponding to the maximally pinned configuration. As a result, the mean velocity of the manifold vanishes exponentially with vanishing driving force according to $v \simeq \exp [-U(F) / T]$, as obtained earlier by a scaling approach [1]. This form for the macroscopic response arises naturally as a result of the cutoff of the algebraic time distribution at the maximal waiting time. We also conjecture a qualitatively different marginal glassy response, with $v \propto\left[\left(F / F_{c}(T)\right]^{1-\mu}\right.$, for a single elastic string and $T>T_{d p}^{s}$. Finally, we discuss how the power-law distribution of waiting times controls the noise spectrum of the vortex density and can therefore be probed experimentally.

This work was supported at Argonne by the U.S. Department of Energy, BES-Material Sciences, under contract No. W-31-109-ENG-38 and at ETH-Zuerich by the Swiss National Foundation. It is a great pleasure to thank L. Ioffe, P. Le Doussal, C. M. Marchetti, and L.W. Chen whose collaboration was crucial and most essential for obtaining the results of this work.

[1] G. Blatter, et al., Rev. Mod. Phys. 66, 1125 (1994).

[2] T. Halpin-Healy and Y-C. Zhang, Phys. Rep. 254, 215 (1995).

[3] V.M. Vinokur, J. Phys. France 471425 (1986).

[4] L. B. Ioffe and V. M. Vinokur, J.Phys. C: Solid State Phys. 20, 6149 (1987).

[5] L. V. Mikheev, B. Drossel, and M. Kardar, Phys. Rev. Lett. 75, 1170 (1995); B. Drossel, J. Stat. Phys 82, 431 (1996).

[6] T. Nattermann, et al, J. Phys. II (France) 2, 1483 (1992).

[7] O. Narayan and D. Fisher, Phys. Rev. B 48, 7030 (1993).

[8] H. A. Makse, A.L. Barabasi, and H. E. Stanley, preprint.

[9] M. Dong, et al, Phys. Rev. Lett. 70, 662 (1993).

[10] H. Kaper, et al, Phys. Rev. Lett. 71, 3713 (1993).

[11] H. Leschorn and L.-H. Tang, Phys. Rev. Lett. 70, 2973 (1993).

[12] H. Leschorn, Physica A 195, 324 (1993).

[13] C. Tang, S. Feng, and L. Golubovic, Phys. Rev. Lett. 72, 1264 (1994).

[14] H. A. Makse and L. A. N. Amaral, Europhys. Lett. 31, 379 (1995).

[15] Y. G. Sinai Theor. Probab. Its Appl. 27247 (1982).

[16] F. Solomon, Ann. Probab. 31 (1975), H. Kesten, M. Koslov, F. Spitzer, Compos. Math. 30 145 (1975)

[17] B. Derrida and Y. Pomeau, Phys. Rev. Lett. 48627 (1982).

[18] B. Derrida J. Stat. Phys. 31433 (1983).

[19] J. P. Bouchaud, A. Comtet, A. Georges and P. Le Doussal, Ann Phys. 201285 (1990) and Europhys. Lett. 3 653 (1987)

[20] M. Feigelman and V. M. Vinokur, J. Phys. France 491731 (1988).

[21] P. Le Doussal, Phys. Rev. Lett. 623097 (1989)

[22] M. Alba, M. Ocio, J. Hammann, Europhys. Lett. 242 (1986).

[23] L.F. Cugliandolo and J. Kurchan, Phys. Rev. Lett. 71173 (1993) and J. Phys. A Math Gen 275749 (1994). 
[24] P. Le Douusal and V. M. Vinokur, Physica C 254, 63 (1995)

[25] H. J. Herrmann and H. E. Stanley, Phys. Rev. Lett. 53, 1121 (1984)

[26] L.H. Tang, T. Nattermann and B. M. Forrest, Phys. Rev. Lett. 652422 (1990).

[27] J. Galambos, The asymptotic theory of extreme order statistics (J. Wiley, New York, 1978). 